\title{
USP11 regulates p53 stability by deubiquitinating p53*
}

\author{
Jia-ying $\mathrm{KE}^{\dagger 1,2}$, Cong-jie DAI ${ }^{1,2}$, Wen-lin WU ${ }^{1,2}$, Jin-hua $\mathrm{GAO}^{3}$, Ai-juan XIA ${ }^{4}$, \\ Guang-ping LIU ${ }^{5}$, Kao-sheng LV ${ }^{6}$, Chun-lin WU $\$$ \\ $\left({ }^{1}\right.$ College of Chemistry and Life Science, Quanzhou Normal University, Quanzhou 36200, China) \\ ( ${ }^{2}$ The Higher Educational Key Laboratory for Molecular Biology and Pharmacology of Fujian Province, Quanzhou 36200, China) \\ ( ${ }^{3}$ Xiamen Women and Children Health Hospital, Xiamen 361005, China) \\ $\left({ }^{4}\right.$ Shouguang People's Hospital, Shouguang 262700, China) \\ ( 5 Department of Orthopedics, Central Hospital of Zibo, Zibo 255000, China) \\ $\left({ }^{6}\right.$ Department of Pathology, University of Chicago, Chicago 60102, Illinois, USA) \\ ('Department of Pathology, the Second Affiliated Hospital of Fujian Medical University, Quanzhou 36200, China) \\ †E-mail: kejiaying2003@163.com; wuchunlin120@sina.com \\ Received June 30, 2014; Revision accepted Nov. 11, 2014; Crosschecked Nov. 26, 2014
}

\begin{abstract}
The p53 tumor suppressor protein coordinates the cellular responses to a broad range of cellular stresses, leading to DNA repair, cell cycle arrest or apoptosis. The stability of p53 is essential for its tumor suppressor function, which is tightly controlled by ubiquitin-dependent degradation primarily through its negative regulator murine double minute 2 (Mdm2). To better understand the regulation of p53, we tested the interaction between p53 and USP11 using co-immunoprecipitation. The results show that USP11, an ubiquitin-specific protease, forms specific complexes with p53 and stabilizes p53 by deubiquitinating it. Moreover, down-regulation of USP11 dramatically attenuated p53 induction in response to DNA damage stress. These findings reveal that USP11 is a novel regulator of p53, which is required for $\mathrm{p} 53$ activation in response to DNA damage.
\end{abstract}

Key words: p53, USP11, Deubiquitination, Stability doi: 10.1631 jzus.B1400180
CLC number: Q291

\section{Introduction}

p53 is mutated in more than $50 \%$ of human cancers, indicating that p53 is a crucial tumor suppressor (Liu et al., 2014). p53 functions as a sequence-specific transcription factor and through a range of target genes involved in cell cycle arrest

\footnotetext{
ॠ Corresponding author

* Project supported by the Key Project of Colleges and Universities in Fujian Province Serving the Construction of the West-strait (No. A101), the Foundation for the 2013 Research Plan of University Key Teacher Domestic Visitor of the Ministry of Education, and the Foundation for the Second Batch of Key Teacher of Quanzhou Normal College (the personnel department of QNC [2012] No. 1), China

(D) ORCID: Jia-ying KE, http://orcid.org/0000-0001-8638-230X; Chun-lin WU, http://orcid.org/0000-0003-1686-8907

(C) Zhejiang University and Springer-Verlag Berlin Heidelberg 2014
}

(such as p21) and apoptosis (such as puma and bax). p53 maintains genomic integrity and prevents cell transformation and tumor formation (Vogelstein et al., 2000; Riley et al., 2008; Kruse and Gu, 2009). Recent studies indicate that the p53 target gene TIGAR (TP53-induced glycolysis and apoptosis regulator), a regulator of glycolysis, also modulates p53 tumor suppressor function (Bensaad et al., 2006; Madan et al., 2012; Won et al., 2012). p53 is kept at very low levels in normal cells and dramatically stabilized in response to a variety of stress signals, including DNA damage, abnormal oncogenic activation, and hypoxia (Harris and Levine, 2005; Kruse and Gu, 2009; Vousden and Prives, 2009). The stability of p53 is primarily regulated by its negative regulator murine double minute 2 (Mdm2), which binds p53 and 
functions as an E3 ubiquitin ligase leading to p53 ubiquitination and degradation (Haupt et al., 1997; Honda et al., 1997; Kubbutat et al., 1997; Nag et al., 2013).

It is well documented that ubiquitination of p53 can be reversed by a number of deubiquitinating enzymes (DUBs). The ubiquitin-specific protease USP7 (also known as HAUSP) deubiquitinates and stabilizes p53 (Li et al., 2002). However, USP7 also deubiquitinates and stabilizes Mdm2, which is in fact a better binding partner of USP7 than p53. A modest reduction of USP7 in cells results in an increase in p53 levels (Cummins et al., 2004), while nearly complete ablation of USP7 causes an almost complete loss of Mdm2 and consequently significant stabilization of p53 and cell death (Cummins et al., 2004; Li et al., 2004). USP10, a cytoplasmic ubiquitin-specific protease, is stabilized after DNA damage and translocates to the nucleus to deubiquitinate p53 and counteracts Mdm2-induced p53 nuclear export and degradation (Yuan et al., 2010). USP29 is another DUB involved in the p53 pathway, which deubiquitinates and stabilizes p53 in response to oxidative stress (Liu et al., 2011). Recently two OTU family members, DUB OTUB1 (the ovarian tumor domaincontaining $\mathrm{Ub}$ aldehyde-binding protein 1) and OTUD5 (OTU deubiquitinase 5), have been shown to positively regulate p53 stability by deubiquitinating p53 (Sun et al., 2012; Luo et al., 2013; Li et al., 2014; Sun and Dai, 2014). Overall, deubiquitination plays an important role in p53 stabilization and activation under both normal and stress conditions.

USP11 is a member of the ubiquitin-specific protease family. USP11 regulates BRCA2 expression levels by deubiquitinating it and participates in the cellular response to DNA damage (Schoenfeld et al., 2004). Recently, USP11 has been shown to deubiquitinate and stabilize promyelocytic leukemia (PML) and inhibit multiple malignant characteristics of brain tumors (Wu et al., 2014). Moreover, USP11 plays a key role in homologous recombination repair of DNA double-strand breaks and may be a novel therapeutic target (Wiltshire et al., 2010). Other substrates deubiquitinated by USP11 include RanBPM (Ideguchi et al., 2002), IкB $\alpha$ (Sun et al., 2010), and type I transforming growth factor $\beta$ (TGF $\beta$ ) receptor ALK5 (Al-Salihi et al., 2012). Here we show that USP11 is a novel positive regulator of $\mathrm{p} 53$, which deubiquitinates and stabilizes p53 and is required for p53 induction in response to DNA damage.

\section{Materials and methods}

\subsection{Plasmid construction}

Full-length complementary DNA (cDNA) encoding USP11 was obtained by polymerase chain reaction (PCR) and subcloned into pCMV-Flag (Clontech). Pfu polymerase (Promega) was used to generate the point mutation of USP11 (USP11 C275S) through a PCR-based site-directed mutagenesis method. All constructs derived from PCR products were verified by DNA sequencing (Invitrogen).

\subsection{Cell culture, transfection, and treatments}

Human HEK293, HEK293T, H1299, and U2OS cells were maintained in Dulbecco's minimal essential medium (Gibco) supplemented with $10 \%$ fetal bovine serum (HyClone), 100 IU penicillin, and $100 \mu \mathrm{g} / \mathrm{ml}$ streptomycin in a $37{ }^{\circ} \mathrm{C}$ incubator at $5 \%$ $\mathrm{CO}_{2}$. Cells were transiently transfected using the standard calcium phosphate method. MG132, cycloheximide (CHX), and etoposide (Sigma) were added to subconfluent cells at the indicated concentrations.

\subsection{RNA interference}

For RNA interference experiments, oligonucleotides targeting USP11 (shUSP11-1 5'-CCAGTGG CGCCAGATAGAA-3') were cloned into lentivirusbased vector pLL3.7. Recombinant lentiviral plasmids and the packaging plasmids VSV-G, RSV-REV, and pMDL were co-transfected into 293T cells. Two days later, the viral supernatants were collected to infect target cells in the presence of $6 \mu \mathrm{g} / \mathrm{ml}$ polybrene (Sigma).

\subsection{Western blotting and immunoprecipitation}

Cell were collected and washed twice with phosphate buffered saline (PBS) and then cell extracts were prepared in Triton X-100 buffer $(20 \mathrm{mmol} / \mathrm{L}$ Tris- $\mathrm{HCl}(\mathrm{pH} 7.5), 150 \mathrm{mmol} / \mathrm{L} \mathrm{NaCl}, 1 \%$ Triton $\mathrm{X}-100,20 \mathrm{mmol} / \mathrm{L} \beta$-glycerophosphate, $10 \mathrm{mmol} / \mathrm{L} \mathrm{NaF}$, $1 \mathrm{mmol} / \mathrm{L}$ phenylmethylsulfonyl fluoride, $1 \mathrm{mmol} / \mathrm{L}$ sodium orthovanadate, $10 \mu \mathrm{g} / \mathrm{ml}$ leupeptin, $2 \mu \mathrm{g} / \mathrm{ml}$ aprotinin, and $1 \mathrm{mmol} / \mathrm{L}$ ethylene diamine tetraacetic acid (EDTA)). The protein samples were boiled for 
5 min in the sodium dodecyl sulfate (SDS) buffer and resolved in the SDS polyacrylamide gelelectrophoresis (PAGE) gel. The proteins separated in the gel were transferred to the polyvinylidene fluoride (PVDF) membrane (Millipore). After blocking with 5\% skim milk for $1 \mathrm{~h}$ at room temperature, the membranes were probed with antibodies at $4{ }^{\circ} \mathrm{C}$ overnight, followed by incubating with horseradish peroxidaseconjugated secondary antibodies (Thermo) for $1 \mathrm{~h}$ at room temperature. The amount of proteins was visualized by chemiluminescence. The antibodies used were as follows: anti-Flag M2 and anti-actin monoclonal antibodies (Sigma); hemagglutinin (HA; Y-11), anti-Myc (9E10), USP11, and p53 (DO1) monoclonal antibodies (Santa Cruz Biotechnology).

For the immunoprecipitation experiments, cell extracts were incubated with indicated antibodies overnight at $4{ }^{\circ} \mathrm{C}$. Cell extracts were then incubated with Protein $\mathrm{G}$ sepharose beads for another $4 \mathrm{~h}$. The beads were centrifuged and washed five times with lysis buffer. The immunocomplexes were boiled for $10 \mathrm{~min}$ in the SDS buffer and subjected to Western blotting analysis.

\subsection{Quantitative real-time PCR}

Total RNA was extracted using the RNA Simple Total RNA Kit (Tiangen Biotech, Beijing, China) following the manufacturer's instructions. One microgram of the total RNA was reverse-transcribed to first-strand cDNA by using the First-Strand cDNA Synthesis Kit (Toyobo, Osaka, Japan). The expression levels of $p 53, p 21$, bax and puma were analyzed by quantitative real-time PCR with Power SYBR Green PCR Master Mix (Applied Biosystems, Foster City, CA, USA) following the manufacturer's instructions on ABI 7500 real-time PCR system (Applied Biosystems). The primers of $p 53, \mathrm{p} 53$ target genes $p 21$, bax, and puma, and glyceraldehyde-3-phosphate dehydrogenase $(G A P D H)$ were as follows: human $p 53$ forward 5'-ACTGCATGGACGATCTGTTG-3' and reverse 5'-GTGACAGGGTCCTGTGCTG-3'; human p21 forward 5'-TTTCAGCCACAGCGACCATG-3' and reverse 5'-AAAGTTCCACCGTTCTCGGG-3'; human bax forward 5'-TGGAGATGAACTGGA CAGCA-3' and reverse 5'-GAAGTTGCCATCAGC AAACA-3'; human puma forward 5'-GGATGGCG GACGACCTC-3' and reverse 5'-CGGGCAAGGCT GGCAGT-3'; human GAPDH forward 5'-GACAT
CAAGAAGGTGGTGAA-3' and reverse 5'-TGTC ATACCAGGAAATGAGC-3'.

\subsection{In vivo ubiquitination assay}

H1299 cells were cotransfected with expression vectors of HA-p53, Myc-ubiquitin, and Flag-USP11. Thirty-six hours after transfection, cells were treated with $5 \mu \mathrm{mol} / \mathrm{L}$ MG132 for $3 \mathrm{~h}$ before being harvested. Then cells were lysed with RIPA buffer $(0.5 \%(5 \mathrm{~g} / \mathrm{L})$ SDS, $0.5 \%(5 \mathrm{~g} / \mathrm{L})$ sodium deoxycholate, $0.5 \%$ Nonidet $\mathrm{P}-40,150 \mathrm{mmol} / \mathrm{L} \mathrm{NaCl}, 10 \mathrm{mmol} / \mathrm{L} \mathrm{NaF}, 20 \mathrm{mmol} / \mathrm{L}$ $\beta$-glycerophosphate, $1 \mathrm{mmol} / \mathrm{L}$ sodium orthovanadate, $1 \mathrm{mmol} / \mathrm{L}$ phenylmethylsulfonyl fluoride, $10 \mu \mathrm{g} / \mathrm{ml}$ leupeptin, and $2 \mu \mathrm{g} / \mathrm{ml}$ aprotinin), followed by incubation with HA antibody overnight at $4{ }^{\circ} \mathrm{C}$ and incubated with Protein $\mathrm{G}$ sepharose beads for another $4 \mathrm{~h}$. The immunoprecipitates were boiled in the SDS buffer and subjected to Western blotting analysis. p53 proteins were immunoprecipitated by HA antibodies and ubiquitinated p53 was detected by anti-Myc antibody.

\section{Results}

\section{1 p53 interacts with USP11}

We detected that the ubiquitin-specific protease USP11 interacts with p53. As shown in Fig. 1a, Flag-USP11 can be observed in the HA-p53 but not in control immunoprecipitates. Since we know p53 is induced rapidly in response to various stresses including DNA damage, we examined the interaction between p53 and USP11 in response to DNA damage caused by the treatment with etoposide. As shown in Fig. 1b, the etoposide treatment significantly enhanced the interaction between endogenous p53 and USP11. These results indicate that $\mathrm{p} 53$ interacts with USP11 specifically in mammalian cells, which is consistent with the previous study of Sowa et al. (2009).

\subsection{USP11 stabilizes p53}

Since USP11 is an ubiquitin-specific protease, we explored the possibility that USP 11 could function to deubiquitinate and stabilize p53. To this end, we overexpressed increasing amounts of Flag-USP11 in U2OS cells. As shown in Fig. 2a, overexpression of USP11 led to a dose-dependent increase in the level of endogenous $\mathrm{p} 53$. We also observed a concomitant 
accumulation of $p 21$, a p53 target gene. Overexpression of USP 11 had no effect on $\mathrm{p} 53$ messenger RNA (mRNA) levels as shown in Fig. 2b, suggesting that USP11 might enhance p53 level by enhancing p53 protein stability. To confirm the role of USP11 in regulating p53 protein levels, we treated U2OS control cells and cells overexpressing USP11 with CHX to examine the stability of p53. As shown in Fig. 2c, the half-life of p53 was prolonged in cells transfected

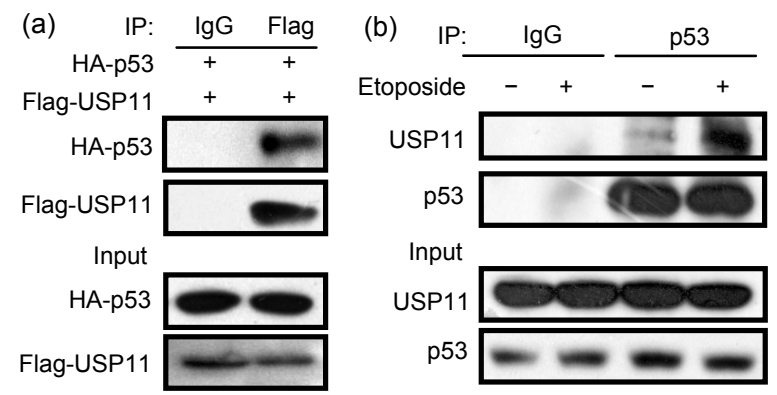

Fig. 1 Interaction of p53 with USP11

(a) HEK293 cells were cotransfected with Flag-USP11 and HA-p53. Cells were collected and lysed $24 \mathrm{~h}$ after transfection. Cell lysates were subjected to immunoprecipitation with anti-Flag antibody and immunoblotted with anti-HA antibody. (b) U2OS cells were treated with or without $20 \mu \mathrm{mol} / \mathrm{L}$ etoposide for $24 \mathrm{~h}$. Cells were harvested and lysed. We adjusted the p53 to the same level and then performed the co-immunoprecipitation assay (a)

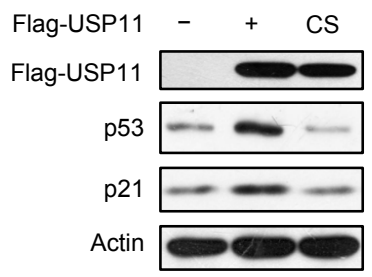

(b)

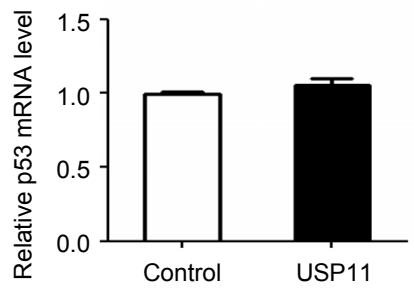

with USP11. These results suggest that USP11 regulates $\mathrm{p} 53$ stability.

\subsection{USP11 deubiquitinates p53}

USP11 can stabilize p53 by deubiquitinating it. We next carried out an in vivo ubiquitination assay to examine whether USP11 diminishes p53 ubiquitination. Constant amounts of HA-p53, Myc-Ub together with Flag-USP11 (wild type or C275S) were cotransfected into H1299 cells. Cells were treated with the proteasome inhibitor MG132 before being harvested to observe p53 ubiquitination. HA antibody immunoprecipitated ubiquitin-conjugated proteins and p53-ubiquitin conjugates were detected by Myc antibody. As Fig. 3a shows, the ubiquitination of p53 was markedly reduced by cotransfection of wild type USP11. We also observed that USP11 C275S, the catalytic-inactive mutant, lost the ability to counteract the ubiquitination of p53. Conversely, down-regulation of USP11 increased endogenous p53 ubiquitination (Fig. 3b). U2OS cells stably expressing USP11 shRNA and the control cells were treated with MG132 before being harvested. As Fig. 3b shows, endogenous p53 ubiquitination was markedly increased in USP11 knockdown cells compared with the control cells. These results suggest that USP11 deubiquitinates $\mathrm{p} 53$, therefore leading to the stabilization of $\mathrm{p} 53$.

(c)

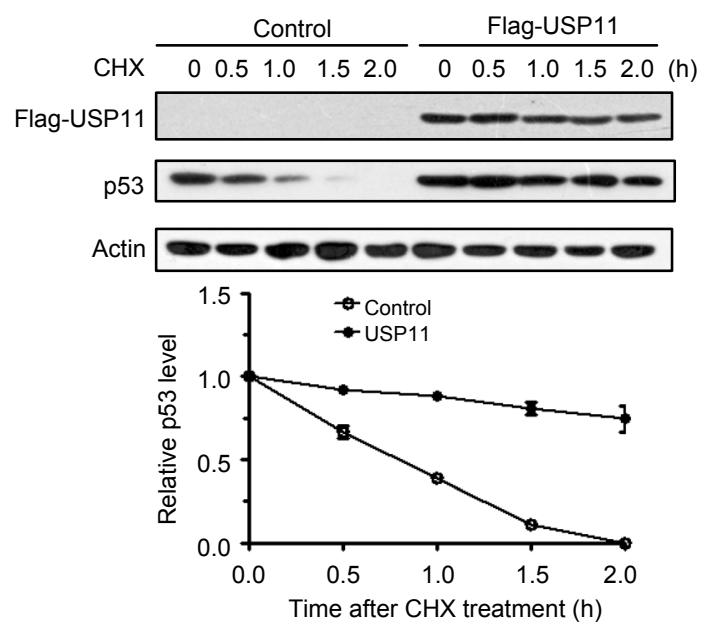

Fig. 2 Regulation of p53 stability by USP11

(a) U2OS cells were transfected with increasing amounts of Flag-USP11. Cell lysates were prepared and analyzed by Western blotting with the indicated antibodies $24 \mathrm{~h}$ post-transfection. (b) p53 mRNA levels of U2OS control cells and USP11-expressing cells were measured by quantitative real-time PCR. Results are shown as mean \pm standard deviation (SD) of triplicate experiments and normalized to the control. (c) Half-life assay of endogenous p53 protein. U2OS cells transfected with vector or Flag-USP11 were treated with $30 \mu \mathrm{g} / \mathrm{ml}$ cycloheximide (CHX) and harvested at the indicated time. Protein levels were detected by Western blotting using the indicated antibodies. The values are obtained from three independent experiments and are normalized to the actin control. For each experimental condition, the signal at the start of the experiment was set to 1 . CS: Cys 275 mutants to Ser 

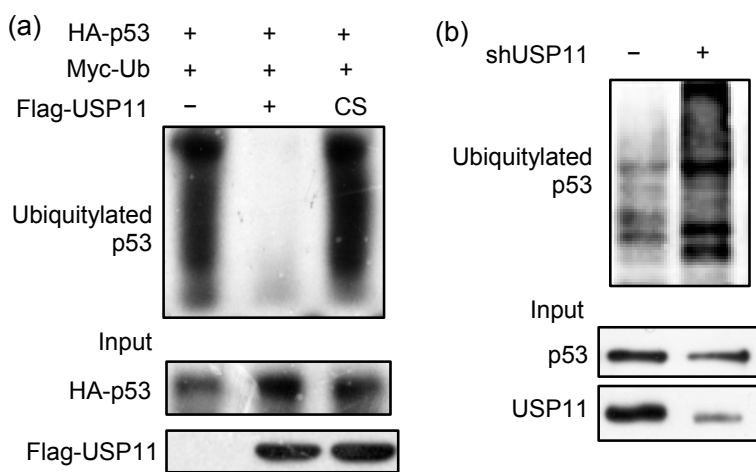

Fig. 3 Deubiquitination of p53 by USP11

(a) H1299 cells transfected with the indicated plasmids were treated with MG132 for $3 \mathrm{~h}$ before being harvested. Cell lysates were incubated with HA antibody and the immunoprecipitates were analyzed by immunoblotting with anti-Myc antibody. (b) U2OS cells stably expressing control or USP11 shRNA were treated with MG132 before being harvested. p53 was immunoprecipitated with anti-p53 DO-1 antibody and immunoblotted with anti-ubiquitin antibody. CS: Cys 275 mutants to Ser

\subsection{USP11 is required for $\mathrm{p53}$ induction in re- sponse to DNA damage}

p53 is induced rapidly in response to stress, so we considered whether USP11 is needed for p53 stabilization and activation in response to a DNA damage signal. U2OS cells stably expressing USP11 shRNA or control cells were treated with etoposide. p53 was dramatically stabilized after treatment of cells with etoposide. However, etoposide-induced p53 stabilization was largely impeded in cells where USP11 was silenced (Fig. 4a). In concert with this result, mRNA expression of p53-inducible genes, such as puma, p21, and bax, was also attenuated in USP11 depletion cells (Fig. 4b). Our data show that USP11 is required for the stabilization and activation of p53 in response to DNA damage.

\section{Discussion}

The tumor suppressor p53 is a short-lived protein and cellular p53 levels are tightly controlled by proteasome-dependent degradation following p53 ubiquitination (Michael and Oren, 2003; Brooks and $\mathrm{Gu}, 2006)$. p53 ubiquitination is primarily achieved by E3 ubiquitin ligase Mdm2, which binds p53 with high affinity and efficiently ubiquitinates p53 (Haupt

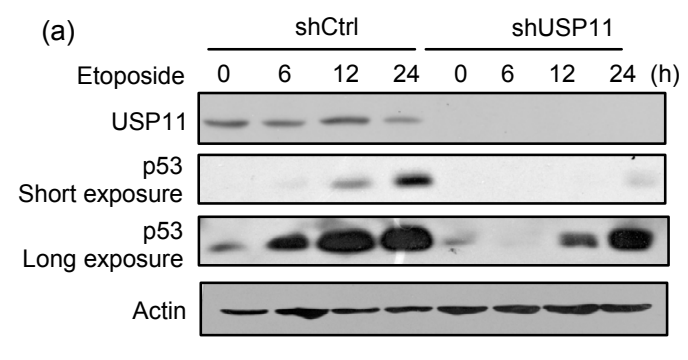

(b)

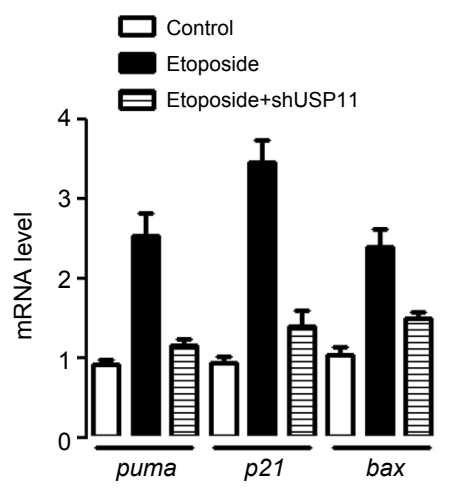

Fig. 4 Role of USP11 on p53 induction in response to DNA damage

(a) U2OS cells stably expressing USP11 shRNA or control cells were treated with $20 \mu \mathrm{mol} / \mathrm{L}$ etoposide for $12 \mathrm{~h}$. Cells were harvested and cell lysates were subjected to immunoblotting analysis with indicated antibodies. (b) Total RNAs were extracted, reverse-transcribed, and analyzed by quantitative real-time PCR for the indicated genes ( $p 21$, bax, and puma). Results are shown as mean $\pm \mathrm{SD}$ of triplicate experiments and normalized to the control

et al., 1997; Honda et al., 1997; Kubbutat et al., 1997). Other E3 ubiquitin ligases have been identified to contribute to the degradation of p53 in Mdm2independent pathways, including COP1, Pirh2, Arf-BP1, TRAF7, and Cullin4B (Leng et al., 2003; Dornan et al., 2004; Chen et al., 2005; Wang et al., 2013; Thirunavukarasou et al., 2014). The ubiquitination of $\mathrm{p} 53$ could be reversed by deubiquitination enzymes. Recently, a number of DUBs have been discovered, which control the extent of p53 ubiquitination through deubiquitinating p53 (Cummins et al., 2004; Yuan et al., 2010; Liu et al., 2011; Sun et al., 2012; Luo et al., 2013).

In this study, we report that USP11 is another DUB that functions to deubiquitinate and stabilize p53. We show that (1) USP11 is a novel p53 interacting protein; (2) USP11 stabilizes p53 by deubiquitinating p53; and, (3) USP11 is required for the stabilization and activation of p53 in response to DNA damage. These results provide new insights into 
the function of USP11 and reveal a novel mechanism of p53 regulation in response to DNA damage.

USP7, USP10, USP42, USP29, and other DUBs regulate p53 ubiquitination levels under selective conditions. USP7 deubiquitinates both $\mathrm{p} 53$ and Mdm2. USP7 prefers to deubiquitinate $\mathrm{Mdm} 2$ in unstressed cells, while Mdm2-USP7 interaction is disrupted through ataxia-telangiectasia-mutated kinase (ATM)-dependent phosphorylation in response to DNA damage, tilting the stabilization of p53 (Meulmeester et al., 2005). USP10 deubiquitinates p53 in the cytoplasm and recycles p53 ubiquitinated by Mdm2. ATM-mediated phosphorylation of USP10 promotes its stabilization and translocation to the nucleus to stabilize and activate p53 following DNA damage (Yuan et al., 2010). Here we show another regulation route of $\mathrm{p} 53$. We found that USP11, similar to USP42, is required to allow the rapid stabilization and activation of $\mathrm{p} 53$ in response to stress. USP11 depletion dramatically inhibits etoposide-induced p53 stabilization (Fig. 4a). It is possible that posttranslational modifications are involved in the regulation of p53-USP11 interaction. USP11 is likely regulated by ATM phosphorylation. Yamaguchi et al. (2007) have shown that USP11 regulates p53 levels via the regulation of IкB kinase- $\alpha$ (IKK- $\alpha$ ) in response to TNF $\alpha$. In our study, we found that USP11 could directly interact with p53 and regulate p53 stability in response to DNA damage. Both modes of regulation could potentially coexist to further fine-tune the control of p53 levels in response to a variety of cellular stresses such as DNA damage or TNF $\alpha$. It has been reported that USP11 could interact with several other DUBs, including USP7, which could stabilize p53 (Sowa et al., 2009). However, the biological function of the interaction between USP7 and USP11 remains unknown. In our studies, we found that knockdown of USP11 largely impeded the activation of $\mathrm{p} 53$. We hypothesize that USP11 might cooperate with USP7 to stabilize p53. In conclusion, our work indicated that USP11 plays an important role in $\mathrm{p} 53$ regulation in response to DNA damage stress.

\section{Compliance with ethics guidelines}

Jia-ying KE, Cong-jie DAI, Wen-lin WU, Jin-hua GAO, Ai-juan XIA, Guang-ping LIU, Kao-sheng LV, and Chun-lin WU declare that they have no conflict of interest.

This article does not contain any studies with human or animal subjects performed by any of the authors.

\section{References}

Al-Salihi, M.A., Herhaus, L., Macartney, T., et al., 2012. USP11 augments TGF $\beta$ signalling by deubiquitylating ALK5. Open Biol., 2(6):120063. [doi:10.1098/rsob. 120063]

Bensaad, K., Tsuruta, A., Selak, M.A., et al., 2006. TIGAR, a p53-inducible regulator of glycolysis and apoptosis. Cell, 126(1):107-120. [doi:10.1016/j.cell.2006.05.036]

Brooks, C.L., Gu, W., 2006. p53 ubiquitination: Mdm2 and beyond. Mol. Cell, 21(3):307-315. [doi:10.1016/j.molcel. 2006.01.020]

Chen, D., Kon, N., Li, M., et al., 2005. ARF-BP1/Mule is a critical mediator of the ARF tumor suppressor. Cell, 121(7): 1071-1083. [doi:10.1016/j.cell.2005.03.037]

Cummins, J.M., Rago, C., Kohli, M., et al., 2004. Tumour suppression: disruption of HAUSP gene stabilizes p53. Nature, 428(6982): 1 p following 486. [doi:10.1038/nature 02501]

Dornan, D., Wertz, I., Shimizu, H., et al., 2004. The ubiquitin ligase COP1 is a critical negative regulator of p53. Nature, 429(6987):86-92. [doi:10.1038/nature02514]

Harris, S.L., Levine, A.J., 2005. The p53 pathway: positive and negative feedback loops. Oncogene, 24(17):2899-2908. [doi:10.1038/sj.onc.1208615]

Haupt, Y., Maya, R., Kazaz, A., et al., 1997. Mdm2 promotes the rapid degradation of p53. Nature, 387(6630):296-299. [doi:10.1038/387296a0]

Honda, R., Tanaka, H., Yasuda, H., 1997. Oncoprotein MDM2 is a ubiquitin ligase $\mathrm{E} 3$ for tumor suppressor p53. FEBS Lett., 420(1):25-27. [doi:10.1016/S0014-5793(97)01480-4]

Ideguchi, H., Ueda, A., Tanaka, M., et al., 2002. Structural and functional characterization of the USP11 deubiquitinating enzyme, which interacts with the RanGTP-associated protein RanBPM. Biochem. J., 367(Pt 1):87-95. [doi:10. 1042/BJ20011851]

Kruse, J.P., Gu, W., 2009. Modes of p53 regulation. Cell, 137(4):609-622. [doi:10.1016/j.cell.2009.04.050]

Kubbutat, M.H., Jones, S.N., Vousden, K.H., 1997. Regulation of p53 stability by Mdm2. Nature, 387(6630):299-303. [doi:10.1038/387299a0]

Leng, R.P., Lin, Y., Ma, W., et al., 2003. Pirh2, a p53-induced ubiquitin-protein ligase, promotes p53 degradation. Cell, 112(6):779-791. [doi:10.1016/S0092-8674(03)00193-4]

Li, M., Chen, D., Shiloh, A., et al., 2002. Deubiquitination of p53 by HAUSP is an important pathway for p53 stabilization. Nature, 416(6881):648-653. [doi:10.1038/nature737]

Li, M., Brooks, C.L., Kon, N., et al., 2004. A dynamic role of HAUSP in the p53-Mdm2 pathway. Mol. Cell, 13(6): 879-886. [doi:10.1016/S1097-2765(04)00157-1]

Li, Y., Sun, X.X., Elferich, J., et al., 2014. Monoubiquitination is critical for ovarian tumor domain-containing ubiquitin aldehyde binding protein 1 (Otub1) to suppress UbcH5 enzyme and stabilize p53 protein. J. Biol. Chem., 289(8): 5097-5108. [doi:10.1074/jbc.M113.533109]

Liu, J., Chung, H.J., Vogt, M., et al., 2011. JTV1 co-activates FBP to induce USP29 transcription and stabilize p53 in 
response to oxidative stress. EMBO J., 30(5):846-858. [doi:10.1038/emboj.2011.11]

Liu, J., Zhang, C., Feng, Z., 2014. Tumor suppressor p53 and its gain-of-function mutants in cancer. Acta Biochim. Biophys. Sin., 46(3):170-179. [doi:10.1093/abbs/gmt144]

Luo, J., Lu, Z., Lu, X., et al., 2013. OTUD5 regulates p53 stability by deubiquitinating p53. PLOS ONE, 8(10): e77682. [doi:10.1371/journal.pone.0077682]

Madan, E., Gogna, R., Kuppusamy, P., et al., 2012. TIGAR induces p53-mediated cell-cycle arrest by regulation of RB-E2F1 complex. Br. J. Cancer, 107(3):516-526. [doi:10. 1038/bjc.2012.260]

Meulmeester, E., Pereg, Y., Shiloh, Y., et al., 2005. ATMmediated phosphorylations inhibit Mdmx/Mdm2 stabilization by HAUSP in favor of p53 activation. Cell Cycle, 4(9):1166-1170. [doi:10.4161/cc.4.9.1981]

Michael, D., Oren, M., 2003. The p53-Mdm2 module and the ubiquitin system. Semin. Cancer Biol., 13(1):49-58. [doi:10.1016/S1044-579X(02)00099-8]

Nag, S., Qin, J., Srivenugopal, K.S., et al., 2013. The MDM2p53 pathway revisited. J. Biomed. Res., 27(4):254-271. [doi:10.7555/JBR.27.20130030]

Riley, T., Sontag, E., Chen, P., et al., 2008. Transcriptional control of human p53-regulated genes. Nat. Rev. Mol. Cell Biol., 9(5):402-412. [doi:10.1038/nrm2395]

Schoenfeld, A.R., Apgar, S., Dolios, G., et al., 2004. BRCA2 is ubiquitinated in vivo and interacts with USP11, a deubiquitinating enzyme that exhibits prosurvival function in the cellular response to DNA damage. Mol. Cell. Biol., 24(17):7444-7455. [doi:10.1128/MCB.24.17.74447455.2004]

Sowa, M.E., Bennett, E.J., Gygi, S.P., et al., 2009. Defining the human deubiquitinating enzyme interaction landscape. Cell, 138(2):389-403. [doi:10.1016/j.cell.2009.04.042]

Sun, W., Tan, X., Shi, Y., et al., 2010. USP11 negatively regulates $\mathrm{TNF} \alpha$-induced $\mathrm{NF}-\mathrm{\kappa B}$ activation by targeting on IкB $\alpha$. Cell. Signal., 22(3):386-394. [doi:10.1016/ j.cellsig.2009.10.008]

Sun, X.X., Dai, M.S., 2014. Deubiquitinating enzyme regulation of the p53 pathway: a lesson from Otub1. World $J$. Biol. Chem., 5(2):75-84.
Sun, X.X., Challagundla, K.B., Dai, M.S., 2012. Positive regulation of p53 stability and activity by the deubiquitinating enzyme Otubain 1. EMBO J., 31(3):576-592. [doi:10.1038/emboj.2011.434]

Thirunavukarasou, A., Singh, P., Govindarajalu, G., et al., 2014. E3 ubiquitin ligase Cullin4B mediated polyubiquitination of p53 for its degradation. Mol. Cell. Biochem., 390(1-2):93-100. [doi:10.1007/s11010-014-1960-3]

Vogelstein, B., Lane, D., Levine, A.J., 2000. Surfing the p53 network. Nature, 408(6810):307-310. [doi:10.1038/ 35042675]

Vousden, K.H., Prives, C., 2009. Blinded by the light: the growing complexity of p53. Cell, 137(3):413-431. [doi:10. 1016/j.cell.2009.04.037]

Wang, L., Zhang, S., Qu, G., et al., 2013. Downregulation of ubiquitin E3 ligase TNF receptor-associated factor 7 leads to stabilization of p53 in breast cancer. Oncol. Rep., 29(1):283-287. [doi:10.3892/or.2012.2121]

Wiltshire, T.D., Lovejoy, C.A., Wang, T., et al., 2010. Sensitivity to poly(ADP-ribose) polymerase (PARP) inhibition identifies ubiquitin-specific peptidase 11 (USP11) as a regulator of DNA double-strand break repair. J. Biol. Chem., 285(19):14565-14571. [doi:10.1074/jbc.M110. 104745]

Won, K.Y., Lim, S.J., Kim, G.Y., et al., 2012. Regulatory role of p53 in cancer metabolism via SCO2 and TIGAR in human breast cancer. Hum. Pathol., 43(2):221-228. [doi:10.1016/j.humpath.2011.04.021]

Wu, H.C., Lin, Y.C., Liu, C.H., et al., 2014. USP11 regulates PML stability to control Notch-induced malignancy in brain tumours. Nat. Commun., 5:3214. [doi:10.1038/ ncomms4214]

Yamaguchi, T., Kimura, J., Miki, Y., et al., 2007. The deubiquitinating enzyme USP11 controls an IкB kinase $\alpha$ (IKK $\alpha$ )-p53 signaling pathway in response to tumor necrosis factor $\alpha(\mathrm{TNF} \alpha)$. J. Biol. Chem., 282(47):3394333948. [doi:10.1074/jbc.M706282200]

Yuan, J., Luo, K., Zhang, L., et al., 2010. USP10 regulates p53 localization and stability by deubiquitinating p53. Cell, 140(3):384-396. [doi:10.1016/j.cell.2009.12.032]

\section{中文椥要:}

\section{本文题目：USP11 通过去泛素化 p53 调控 p53 稳定性 USP11 regulates p53 stability by deubiquitinating p53}

研究目的: 深入研究 p53 的泛素化及稳定性的调控。

创新要点: 发现一个新的调控 p53 去泛素化的酶 USP 11 , 它可以通过与 p53 的结合去泛素化并稳定 p 53, 从而揭示了一个新的 p53 去泛素化调控的机制。

研究方法: 通过免疫共沉淀发现 p53 可以与 USP11 结合（图 1a），通过泛素化检测试验发现 USP11 可以 去泛素化 p53（图 3a 和 3b)，最后通过逆转录-聚合酶链式反应（RT-PCR）试验发现在 DNA 损伤后, USP11 对 p53 转录活性的提高是非常重要的。

重要结论: USP11 可通过去泛素化 p53 来调控 p53 稳定性。

关键词组: p53 基因; USP11; 泛素化; 稳定性 